\title{
Comparative phylogeography of Trypanosoma rangeli and Rhodnius (Hemiptera: Reduviidae) supports a long coexistence of parasite lineages and their sympatric vectors
}

\author{
F. MAIA DA SILVA, A. C. V. JUNQUEIRA,+M. CAMPANER, A. C. RODRIGUES, ${ }^{*}$ G. CRISANTE, \\ L. E. RAMIREZ,§Z. C. E. CABALLERO,IIF. A. MONTEIRO,†J. R. COURA,†N. AÑZZ and \\ M. M. G. TEIXEIRA* \\ *Departamento de Parasitologia, Instituto de Ciências Biomédicas, Universidade de Sao Paulo, São Paulo, SP, 05508-900, Brazil, \\ +Departamento de Medicina Tropical, Instituto Oswaldo Cruz, FIOCRUZ, Rio de Janeiro, RJ, Brazil, †Facultad de Ciencias, \\ Departamento de Biologia, Universidad de Los Andes, Mérida, Venezuela, §Departamento de Ciências Biológicas, Parasitologia, UFTM, \\ Uberaba, MG, Brazil, IIInstituto de Medicina Tropical de São Paulo, Universidade de São Paulo, São Paulo, SP, Brazil
}

\begin{abstract}
To make reliable interpretations about evolutionary relationships between Trypanosoma rangeli lineages and their insect vectors (triatomine bugs of the genus Rhodnius) and, thus, about the determinant factors of lineage segregation within $T$. rangeli, we compared phylogenies of parasite isolates and vector species. Sixty-one T. rangeli isolates from invertebrate and vertebrate hosts were initially evaluated in terms of polymorphism of the spliced-leader gene (SL). Further analysis based on SL and SSUrRNA sequences from 33 selected isolates, representative of the overall phylogenetic diversity and geographical range of $T$. rangeli, supported four phylogenetic lineages within this species. By comparing the phylogeny of Rhodnius species with that inferred for T. rangeli isolates and through analysis of the geographical range of the isolates, we showed that there is a very significant overlap in the distribution of Rhodnius species and T. rangeli lineages. Congruence between phylogeographical analysis of both $T$. rangeli lineages and complexes of Rhodnius species are consistent with the hypothesis of a long coexistence of parasites and their vectors, with lineage divergence associated with sympatric species of Rhodnius apparently without association with particular vertebrate hosts. Separation of $T$. rangeli isolates from vectors of distinct complexes living in sympatry favours the absence of gene flow between the lineages and suggests evolution of $T$. rangeli lineages in independent transmission cycles, probably associated to specific Rhodnius spp. ecotopes. A polymerase chain reaction assay based on SL intergenic sequences was developed for simultaneous identification and lineage genotyping of $T$. rangeli in epidemiological surveys.
\end{abstract}

Keywords: evolution, phylogeography, Rhodnius, ribosomal gene, spliced-leader gene, Trypanosoma rangeli

Received 4 March 2007; revision received 4 March 2007; accepted 12 April 2007

\section{Introduction}

The family Trypanosomatidae (Euglenozoa: Kinetoplastida) comprises eight genera of protozoan parasites of vertebrates, invertebrates and plants. The genus Trypanosoma harbours parasites of all vertebrate classes and the life cycle of these

Correspondence: Marta M. G. Teixeira, Fax: +55 1130914717; E-mail: mmgteix@icb.usp.br parasites involves alternation between two hosts: vertebrates and diverse haematophagous invertebrates (vectors). This genus includes only three Trypanosoma species that infect man: T. cruzi and T. rangeli in Latin America and T. brucei in Africa (Stevens et al. 2001).

Trypanosoma rangeli infects humans, and domestic, and sylvatic mammals from Central America to southern South America sharing with T. cruzi the same mammalian hosts and triatomine vectors in overlapping areas. A high 
prevalence of $T$. rangeli human infections has been reported in Central America and northwest South America (D'Alessandro \& Saravia 1999; Guhl \& Vallejo 2003). In Brazil, only three human cases have been reported in Amazonia (Coura et al. 1996), whereas infection of sylvatic mammals and triatomines is very common in this region (Miles et al. 1983; Maia da Silva et al. 2004a, b) and has also been reported in southern, southeastern and central regions (Steindel et al. 1991; Ramirez et al. 2002; Gurgel-Goncalves et al. 2004).

In contrast to T. cruzi and T. brucei, T. rangeli is harmless to mammalian hosts but may cause pathogenicity to its insect vectors in which it can induce difficulties or even lethal effect in moulting and feeding. Unlike T.cruzi whose development in triatomines is entirely restricted to the gut, $T$. rangeli multiplies in the gut but completes its development in the insect salivary gland, where metacyclogenesis takes place as T. brucei in tsetse (Añez 1984; Guhl \& Vallejo 2003). Genetic distances concur with life-cycle differences between T. cruzi and T. rangeli (Stevens et al. 1999; Maia da Silva et al. 2004b). Natural T. rangeli infection appears to be restricted to the gut in all other triatomine genera except Rhodnius, and its transmission through saliva inoculation during probing and feeding has been proved only for species of this genus. Isolates of $T$. rangeli of distinct geographical origin show variable behaviour in different Rhodnius spp., and transmission by bite is mostly restricted to local vector species, suggesting a tight evolutionary relationship between T. rangeli isolates and their sympatric vectors ( $\mathrm{D}^{\prime}$ Alessandro \& Saravia 1999; Guhl \& Vallejo 2003; Vallejo et al. 2003; De Stefani Marquez et al. 2006).

Several host-parasite associations have been investigated by examining the degree of congruence between host and parasite phylogenies. However, much less information is available regarding the co-evolutionary process in protozoan parasites than in ectoparasites and parasitic worms (Page 1991; Paterson \& Banks 2001; Banks \& Paterson 2005; Brooks \& Ferrao 2005; Huyse et al. 2005). Phylogenetic analyses used to address evolutionary processes within Trypanosoma indicate host-parasite associations between the following trypanosomes and their insect vectors: (i) $T$. brucei and related species and the tsetse fly; (ii) T. theileri of artiodactyls and tabanids; (iii) T. lewisi and allied species of rodents and fleas; and (iv) T. cruzi and T. rangeli and triatomine bugs (Stevens et al. 2001; Maia da Silva et al. 2004b; Rodrigues et al. 2006).

The triatomine vectors of $T$. rangeli belong to the tribe Rhodniini (Reduvidae: Triatominae), which comprises two genera of blood-sucking bugs: Rhodnius and Psammolestes. Triatomines of the genus Rhodnius have a complex biogeographical history and a long history of co-evolution with palm trees in Latin America. Species of this genus are primarily associated with palm tree crowns; the distribution of the sylvatic species coincides with the distribution of palms and may be associated with particular niches in distinct palms. The genus Psammolestes comprises species that live in nests of birds. In contrast, triatomines of the genera Triatoma and Panstrongylus have evolved predominantly associated with terrestrial habitats such as rocks, animal burrows and hollow trees (Gaunt \& Miles 2000).

The species of the genus Rhodnius are distributed into three complexes according to phylogenies based on zymodemes (Dujardin et al. 1999) and sequences of 16S mitochondrial rDNA, cytochrome $b$ (cyt $b$ ) and 28S nuclear rRNA (Lyman et al. 1999; Hypsa et al. 2002; Monteiro et al. 2000, 2003; Paula et al. 2006). The Rhodnius complexes are associated with sympatric palms and have different phylogeographical structures as follows: (i) R. pallescens complex formed by $R$. pallescens, $R$. colombiensis and $R$. ecuadoriensis, distributed from the west of the Andes Mountains to Central America, northern and northwest South America; (ii) $R$. brethesi complex, constituted by $R$. brethesi and R. pictipes, R. amazonicus and R. stali, which are restricted to the Amazon region east of the Andes; and (iii) $R$. prolixus complex, formed by $R$. prolixus, $R$. robustus, $R$. neglectus, $R$. nasutus, $R$. domesticus and $R$. neivai. $R$. prolixus is the most widespread species with high prevalence in Central and northwest South America, west of the Andes. $R$. pictipes and $R$. robustus are restricted to north and northwest South America. R. neglectus, R. nasutsus and R. domesticus occur in the northeast and southeast of Brazil (Galvão et al. 2003). The genus Rhodnius includes three domiciled species, $R$. prolixus, R. pallescens and R. ecuadoriensis. R. robustus in Venezuela can enter dwellings and the peridomicile without becoming domestic, whereas in Brazil, $R$. robustus, R. pictipes and $R$. brethesi are considered entirely sylvatic, although there have been reports of these species inside houses and/ or attacking humans (Dujardin et al. 1991; Coura et al. 1996).

A peculiar organization of the spliced-leader (SL) RNA repeats displaying the highly conserved $5 S$ rRNA gene inserted into the SL intergenic region was observed in $T$. rangeli and few other trypanosomatids (Aksoy et al. 1992; Stevens et al. 1999; Gibson et al. 2000). SL sequences are valuable tools for assessing polymorphism and genetic relatedness among closely related species and are useful as taxonomic and diagnostic tools for genera, species and lineages of trypanosomatids (Souto et al. 1996; Teixeira et al. 1996; Zingales et al. 1998; Serrano et al. 1999; Ventura et al. 2001). Intra-specific polymorphisms of the SL gene revealed different groups of $T$. rangeli isolates (Maia da Silva et al. 1999; Grisard et al. 1999; Urrea et al. 2005). The existence of two groups within $T$. rangeli was also indicated by other molecular markers in addition to SL: random amplified polymorphic DNA (RAPD) patterns (Steindel et al. 1994), kDNA (Vallejo et al. 2002, 2003; Urrea et al. 2005) and histone H2A gene (Cuervo et al. 2006). In our previous studies (Maia da Silva et al. 2004a, b) besides confirming the existence of these two lineages, we demonstrated using RAPD 
analysis and phylogeny based on small subunit (SSU) and ITSrDNA (Maia da Silva et al. 2004a, b) that T. rangeli populations are much more complex than previously supposed and are segregated into at least four phylogenetic lineages.

Analysis of a larger number of isolates from different hosts and geographical regions, utilization of additional molecular markers and association of molecular data with ecogeographical and biological traits is required to make reliable interpretations about lineage segregation within $T$. rangeli and evolutionary relationships between parasite lineages and different species of Rhodnius. In this study, we analysed polymorphism of the SL and ribosomal genes of $T$. rangeli by genotyping 61 isolates, and in addition, we inferred phylogenetic relationships among 26 isolates with the following aims: (i) to assess the consistency of $T$. rangeli lineages through analysis of a large collection of isolates; (ii) to confirm the concordance between lineage genotyping and phylogeny using both spliced-leader and ribosomal markers; (iii) to evaluate evolutionary aspects of $T$. rangeli by phylogeographical analysis and by comparing vector and parasite phylogenies; and (iv) to define SL sequences suitable for simultaneous diagnosis and genotyping of T. rangeli.

\section{Materials and methods}

Isolation of Trypanosma rangeli from triatomines and identification and phylogeny of Rhodnius spp.

Triatomines were collected from palm trees in peridomestic and sylvatic environments in Brazil, Venezuela, and Panama.
Triatomines were initially identified morphologically according to Lent \& Wygodzinsky (1979) (Table 1). Fieldcollected triatomines were dissected and their gut and salivary glands removed, gently squeezed onto glass slides containing phosphate-buffered saline, examined under a phase microscope, and samples positive for Trypanosoma rangeli were inoculated separately in biphasic medium for parasite isolation. To obtain pure cultures of T. rangeli, specimens of Rhodnius robustus from a laboratory colony were infected through intracoelomic inoculation of mixed cultures of T. rangeli and Trypanosoma cruzi. After $\sim 30$ days, the haemolymph and salivary glands were examined for the presence of trypanosomes, and the positive samples were inoculated in biphasic medium (Maia da Silva et al. 2004a).

DNA from the same insect specimens from which T. rangeli had been isolated was purified according to Aljanabi \& Martinez (1997), and a 682-bp fragment of the mitochondrial cyt $b$ gene was amplified, cloned and sequenced as before (Monteiro et al. 2003). Two cyt $b$ sequences showing small polymorphisms compared to previously reported sequences of Rhodnius spp. were deposited in GenBank (Accession numbers in parentheses): $R$. robustus II, 365 from Rondonia, Brazil (EF071583); and R. pallescens, 366 from Panamá (EF071584). Sequences obtained from $R$. prolixus and $R$. robustus I from Venezuela were almost identical to those previously described in Monteiro et al. (2003). These sequences were aligned with those from reference species of Rhodnius: R. brethesi (AF045714); R. pictipes (AF045713); R. pallescens (AF045720); R. ecuadoriensis (AF045715); $R$. robustus I (AF421340); R. robustus II (AF421341); R. robustus III (AF421342); R. robustus IV (AF421343); R. prolixus

Table 1 Trypanosoma rangeli isolates, geographical and host species of origin, genotyping using SL, SL and SSUrDNA sequences employed in the phylogenetic analysis

\begin{tabular}{|c|c|c|c|c|c|c|c|}
\hline \multirow[b]{2}{*}{ TryCC +} & \multirow[b]{2}{*}{ Isolate } & & \multirow[b]{2}{*}{ Host species } & \multirow[b]{2}{*}{ Geographical origin $\ddagger$} & \multirow{2}{*}{$\begin{array}{l}\text { TraSL } \\
\text { PCR§ } \\
\text { (bp) }\end{array}$} & \multicolumn{2}{|c|}{ GenBank Accession no. } \\
\hline & & & & & & SL & SSUrDNA \\
\hline \multicolumn{8}{|c|}{ Lineage A } \\
\hline 031 & S. Augustin (SA) & Human & Homo sapiens & Colombia & 417 & M62864 & AJ012417 \\
\hline 020 & Macias & Human & Homo sapiens & Colombia & 417 & - & AJ012415 \\
\hline 024 & H8GS & Human & Homo sapiens & Honduras & 417 & AF083351 & AY491744 \\
\hline 591 & SMH-03 & Human & Homo sapiens & Guatemala & 417 & - & AY491739 \\
\hline 594 & SMH-79 & Human & Homo sapiens & Guatemala & 417 & - & AY491740 \\
\hline 529 & MHOM/VE/99/CH-99 & Human & Homo sapiens & Venezuela (Barinas) & 417 & - & AY491742 \\
\hline $\boldsymbol{\Delta} 530$ & MHOM/VE/99/D-99 & Human & Homo sapiens & Venezuela (Trujillo) & 417 & - EF071551 & - \\
\hline 533 & MAN/VE/00/LOBITA & Dog & Canis familiaris & Venezuela (Barinas) & 417 & - EF071552 & - EF071572 \\
\hline 220 & AT-AEI & Monkey & Saimiri sciureus & Brazil (PA) Marajó@ ${ }^{\circledR}$ & 417 & - EF071553 & AY491747 \\
\hline 202 & AT-ADS & Monkey & Saimiri sciureus & Brazil (PA) Marajó & 417 & - & AY491746 \\
\hline 353 & Maloch-05 & Monkey & Callicebus m. cupreus & Brazil (AC) P. de Castro & 417 & - & AY491750 \\
\hline 369 & ROma 01 & Opossum & Didelphis marsupialis & Brazil (RO) Monte Negro & 417 & - EF071554 & AY491748 \\
\hline 382 & ROma 06 & Opossum & Didelphis marsupialis & Brazil (RO) Monte Negro & 417 & - & AY491749 \\
\hline $\mathbf{\Delta}^{*}$ & P02 & Opossum & Didelphis marsupialis & Brazil (MG) Uberaba & 417 & - & - \\
\hline $\mathbf{\Delta}^{*}$ & $\mathrm{P} 07$ & Opossum & Didelphis marsupialis & Brazil (MG) Uberaba & 417 & - & - \\
\hline $\mathbf{\Delta}^{*}$ & P18 & Opossum & Didelphis marsupialis & Brazil (MG) Uberaba & 417 & - & - \\
\hline $\mathbf{\Delta}^{*}$ & P19 & Opossum & Didelphis marsupialis & Brazil (MG) Uberaba & 417 & - EF071555 & - EF071573 \\
\hline
\end{tabular}




\begin{tabular}{|c|c|c|c|c|c|c|c|}
\hline \multirow[b]{2}{*}{ TryCC +} & \multirow[b]{2}{*}{ Isolate } & & \multirow[b]{2}{*}{ Host species } & \multirow[b]{2}{*}{ Geographical origin $\ddagger$} & \multirow{2}{*}{$\begin{array}{l}\text { TraSL } \\
\text { PCR§ } \\
\text { (bp) }\end{array}$} & \multicolumn{2}{|c|}{ GenBank Accession no. } \\
\hline & & & & & & SL & SSUrDNA \\
\hline $\boldsymbol{\Delta}^{*}$ & P21 & Opossum & Didelphis marsupialis & Brazil (MG) Uberaba & 417 & - EF071556 & - EF071574 \\
\hline 021 & Choachi & Triatomine & Rhodnius prolixus & Venezuela & 417 & - EF071557 & AJ012414 \\
\hline 022 & Palma-2 & Triatomine & Rhodnius prolixus & Venezuela & 417 & - EF071558 & AY491741 \\
\hline $\mathbf{\Delta} 775$ & $\mathrm{VE} / 9$ & Triatomine & Rhodnius prolixus & Venezuela (Barinas) & 417 & - EF071560 & - EF071575 \\
\hline $\mathbf{\Delta} 795$ & $\mathrm{VE} / 3$ & Triatomine & Rhodnius robustus I & Venezuela (Trujillo) & 417 & - EF071559 & - EF071576 \\
\hline$\Delta 1013$ & IRHO/VE/04/F45-04 & Triatomine & Rhodnius prolixus & Venezuela (Barinas) & 417 & - & - \\
\hline$\Delta 1024$ & IRHO/VE/05/Apure 1 & Triatomine & Rhodnius prolixus & Venezuela (Apure) & 417 & - & - \\
\hline$\Delta 1025$ & IRHO/VE/04/F24-04 & Triatomine & Rhodnius prolixus & Venezuela (Barinas) & 417 & - & - \\
\hline $\mathbf{\Delta} 677$ & ROR-20 & Triatomine & Rhodnius robustus II & Brazil (RO) Monte Negro & 417 & - EF071561 & - EF071577 \\
\hline$\Delta 681$ & ROR-68 & Triatomine & Rhodnius robustus II & Brazil (RO) Monte Negro & 417 & - & - \\
\hline$\Delta 683$ & ROR-67 & Triatomine & Rhodnius robustus II & Brazil (RO) Monte Negro & 417 & - & - \\
\hline$\Delta 701$ & ROR-62 & Triatomine & Rhodnius robustus II & Brazil (RO) Monte Negro & 417 & - EF071562 & - EF071578 \\
\hline$\Delta 704$ & ROR-85 & Triatomine & Rhodnius robustus II & Brazil (RO) Monte Negro & 417 & - EF071563 & - EF071579 \\
\hline \multicolumn{8}{|c|}{ Lineage B } \\
\hline 086 & AM80 & Human & Homo sapiens & Brazil (AM) Rio Negro \# & 380 & - EF071547 & AY491766 \\
\hline 261 & AM11 & Human & Homo sapiens & Brazil (AM) Rio Negro & 380 & & AY491758 \\
\hline 207 & AE-AAA & Monkey & Cebuella pygmaea & Brazil (AC) Rio Branco & 380 & - EF071564 & AY491752 \\
\hline 194 & $\mathrm{AE}-\mathrm{AAB}$ & Monkey & Cebuella pygmaea & Brazil (AC) Rio Branco & 380 & - EF071565 & AY491753 \\
\hline 233 & $4-30$ & Monkey & Saguinus l. labiatus & Brazil (AC) Rio Branco & 380 & - & AY491756 \\
\hline 238 & $5-31$ & Monkey & Saguinus l. labiatus & Brazil (AC) Rio Branco & 380 & - & AY491754 \\
\hline 236 & $8-34$ & Monkey & Saguinus f. weddelli & Brazil (AC) Rio Branco & 380 & - & AY491755 \\
\hline 205 & M-12229 & Monkey & Aotus sp & Brazil (AM) Manaus & 380 & - & AY491757 \\
\hline 416 & 2495 & Monkey & Alouatta stramineus & Brazil (AM) Rio Negro & 380 & - EF071566 & AY491760 \\
\hline 427 & 2570 & Monkey & Callicebus lugens & Brazil (AM) Rio Negro & 380 & - & AY491751 \\
\hline 012 & Saimiri & Monkey & Saimiri sciureus & Brazil (AM) Manaus & 380 & - EF071550 & AY491768 \\
\hline 013 & Preguici & Sloth & Choloepus didactylus & Brazil (PA) Belém & 380 & - EF071549 & AY491767 \\
\hline 010 & Legeri & Anteater & Tamandua tetradactyla & Brazil (PA) Belém & 380 & - EF071548 & AY491769 \\
\hline 032 & Legeri & Anteater & Tamandua tetradactyla & Brazil (PA) Belém & 380 & - & AY491759 \\
\hline $\mathbf{\Delta}^{*}$ & 4176 & Triatomine & Rhodnius brethesi & Brazil (AM) Rio Negro & 380 & • EF071567 & - EF071580 \\
\hline $\mathbf{\Delta} 759$ & 4167 & Triatomine & Rhodnius brethesi & Brazil (AM) Rio Negro & 380 & - & - \\
\hline$\Delta 760$ & 4166 & Triatomine & Rhodnius brethesi & Brazil (AM) Rio Negro & 380 & - & - \\
\hline \multicolumn{8}{|c|}{ Lineage C } \\
\hline 014 & PG & Human & Homo sapiens & Panama & 480 & - EF071568 & AJ012416 \\
\hline 328 & 1625 & Human & Homo sapiens & El Salvador & 480 & • EF071569 & AY491738 \\
\hline$\&$ & $B g-60$ & Human & Homo sapiens & Costa Rica & 480 & & X62675 \\
\hline$\&$ & T. leeuwenhoeki & Sloth & Choloepus didactylus & Panama & 480 & AJ012420 & AJ012412 \\
\hline$\&$ & RGB & Dog & Canis familiaris & Colombia & 480 & AJ01419 & AJ009160 \\
\hline$\Delta 1249$ & $\mathrm{~Pa} 482 \mathrm{TD}$ & Triatomine & Rhodnius pallescens & Panama & 480 & - & - \\
\hline$\Delta 1250$ & $\mathrm{~Pa} 476 \mathrm{TD}$ & Triatomine & Rhodnius pallescens & Panama & 480 & - & - \\
\hline$\Delta 1252$ & $\mathrm{~Pa} 480 \mathrm{TD}$ & Triatomine & Rhodnius pallescens & Panama & 480 & - & - \\
\hline$\Delta 1254$ & $\mathrm{~Pa} 487 \mathrm{GS}$ & Triatomine & Rhodnius pallescens & Panama & 480 & - & - \\
\hline A 1260 & Pa 479GS & Triatomine & Rhodnius pallescens & Panama & 480 & - & - \\
\hline $\mathbf{\Delta}^{*}$ & SO18 & Triatomine & Rhodnius pallescens & Colombia (Sucre) & 480 & - & - \\
\hline $\mathbf{\Delta}^{*}$ & $\mathrm{SO} 29$ & Triatomine & Rhodnius pallescens & Colombia (Sucre) & 480 & - EF071570 & - EF071581 \\
\hline $\mathbf{\Delta}^{*}$ & G5 & Triatomine & Rhodnius pallescens & Colombia (Sucre) & 480 & • EF071571 & - EF071582 \\
\hline \multicolumn{8}{|c|}{ Lineage D } \\
\hline 023 & SC58 & Rodent & Echimys dasythrix & Brazil (SC) & 500 & AF083350 & AY491745 \\
\hline
\end{tabular}

†TryCC, number codes of cultures cryopreserved in the Trypanosomatid Culture Collection of the Department of Parasitology, University of São Paulo; $\boldsymbol{\Delta}$ new isolates characterized in this study; *only DNA samples were available; \& only sequences from GenBank were employed in this study; • sequences obtained in this study and deposited in the GenBank.

‡Geographical origin of the isolates [country (state) city]: @, Marajó Island; \#, Rio Negro region. Brazilian states: MG, Minas Gerais; RO, Rondônia; AC, Acre; PA, Para; AM; Amazonas; SC, Santa Catarina.

§DNA amplified fragments (in bp) generated by TraSL-PCR assay from DNA of T. rangeli isolates. 
(AF045718); R. neglectus (AF045716). Sequences of Triatoma infestans (AF045721), Triatoma sordida (AF045730) and Triatoma dimidiata (AF045726) were used as outgroups for Rhodniini. Alignment of cyt $b$ sequences was performed using the program GENEDOC and manually refined. Maximum parsimony (MP) analysis was performed using a heuristic search strategy and the default options of PAUP* $4.0 \mathrm{~b} 10$ (Swofford 2002). Bootstrapped MP analysis with 100 replicates was done using PAUP with parameters settings as described in Rodrigues et al. (2006).

\section{Growth, identification and genotyping of T. rangeli isolates}

Sixty-one T. rangeli isolates from distinct host species and geographical areas were used in this study, including 27 new isolates (Table 1). Trypanosome cultures and DNA extraction were performed as previously described (Maia da Silva et al. 2004a). Besides morphological identification, the new isolates were also tested using a $T$. rangeli-specific polymerase chain reaction (PCR) assay (Maia da Silva et al. 2004a). All new T. rangeli isolates were also genotyped by PCR amplification of ITS rDNA as before (Maia da Silva et al. 2004b).

\section{PCR amplification of the whole SL repeat units}

The location and sequences of the primers employed for PCR amplification of SL gene sequences are shown in Fig. 1. Whole SL repeats of T. rangeli were amplified using primers RSL1 and RSL2 (Fig. 1b) in $25-\mu \mathrm{L}$ reaction mixtures containing $200 \mu \mathrm{M}$ of each dNTP, $20 \mu \mathrm{M}$ of each primer, $50 \mathrm{ng}$ of DNA templates and 2.5 U of Taq DNA polymerase. Reactions were cycled 30 times as follows: $1 \mathrm{~min}$ at $94{ }^{\circ} \mathrm{C}$, $2 \mathrm{~min}$ at $48^{\circ} \mathrm{C}$ and $2 \mathrm{~min}$ at $72{ }^{\circ} \mathrm{C}$ (with an initial cycle of 3 min at $94^{\circ} \mathrm{C}$ and a final cycle of $10 \mathrm{~min}$ at $72^{\circ} \mathrm{C}$ ). Amplified products were separated in a $2 \%$ agarose gel, stained with ethidium bromide, transferred to nylon membranes and hybridized with the SL201 probe complementary to T. cruzi SL exon sequence (Teixeira et al. 1996).

\section{Standardization of TraSL-PCR for T. rangeli-specific amplification of SL intergenic-spacer sequences}

Primers TraSL1 and TraSL2 were designed for PCR amplification (TraSL-PCR) of a $T$. rangeli-specific DNA fragment of $\sim 380-500 \mathrm{bp}$ of the intergenic spacer, which is flanked by the SL intron and $5 \mathrm{~S}$ rRNA. These oligonucleotides are complementary to intron (TraSL1) or 5S rRNA (TraSL2) sequences, which are highly conserved within $T$. rangeli (Fig. 1c). Amplification reactions were prepared as described above for the complete repeat of the SL gene and cycled 30 times as follows: $1 \mathrm{~min}$ at $94^{\circ} \mathrm{C}, 1 \mathrm{~min}$ at $68^{\circ} \mathrm{C}$ and $1 \mathrm{~min}$ at $72{ }^{\circ} \mathrm{C}$ (with an initial cycle of $3 \mathrm{~min}$ at $94^{\circ} \mathrm{C}$ and a final cycle of $10 \mathrm{~min}$ at $72{ }^{\circ} \mathrm{C}$ ). PCR products were electrophoresed in $2.5 \%$ agarose gel and stained with ethidium bromide.

Sequencing and data analysis of SL and SSU rDNA gene sequences

PCR-amplified whole SL repeats and SL intergenic sequences of $T$. rangeli isolates were purified from agarose gels and cloned, and at least three clones from each isolate were sequenced. Sequences corresponding to variable region of V7-V8 flanked by conserved sequences of SSUrRNA ( $\sim 900 \mathrm{bp})$ from 11 new $T$. rangeli isolates were determined in this study and deposited in the GenBank (Table 1). These sequences were aligned with those from other isolates retrieved from GenBank, most previously determined by our group (Table 1) and used to infer phylogenies using the MP and maximum likelihood (ML) methods.

Alignments of SL and SSUrRNA sequences were done using GENEDOC with final adjustments by eye. MP analysis was performed using the default options of PAUP 4.0b10. ML parameters were optimized using the hierarchical likelihood test in MODeltest 3.06 (Posada \& Crandall 1998) and ML analysis was carried out using TREEPUZzLE 5.0 (Strimmer \& Von Haeseler 1996). Bootstrap analyses with 100 replicates were performed for MP and ML phylogenies as before (Rodrigues et al. 2006). The program TREEMAP 2.02 (Page \& Charleston 1998) was used to identify cophylogenetic events between Rhodnius spp. and T. rangeli lineages. Alignments used in this study are available from the authors upon request.

\section{Results}

Identification and genotyping of new T. rangeli isolates

Sixty-one Trypanosoma rangeli isolates were examined in this study, including 32 isolates that we had studied previously using other molecular markers (Maia da Silva et al. 2004a, b) and 27 new isolates, with most (21) from sylvatic, peridomestic and domestic species of Rhodnius spp. from Venezuela, Colombia, Panama and Brazil (Table 1). Isolates investigated in this study showed morphology and behaviour in mice and triatomines compatible with $T$. rangeli (data not shown), and were selected after species confirmation using a T. rangeli-specific PCR assay (Maia da Silva et al. 2004a). All new $T$. rangeli isolates were genotyped using internal transcribed spacer (ITS) ribosomal markers and ascribed to previously defined lineages as before (Maia da Silva et al. 2004b): (i) isolates from the Brazilian Amazon region were genotyped as lineages A or B; (ii) Isolates from Venezuela and southeastern Brazil were ascribed to lineage A; (iii) isolates from Panama and Colombia were all genotyped as lineage $\mathrm{C}$. 
(a)

(c)

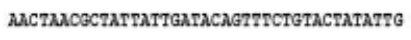

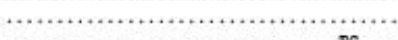

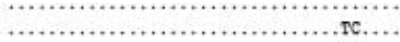
(............................... ……...............................

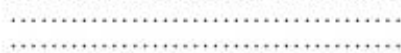

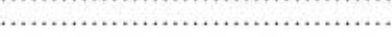
leeuvenhoek: $5 \mathrm{C} 58$ 7. crusi (b)

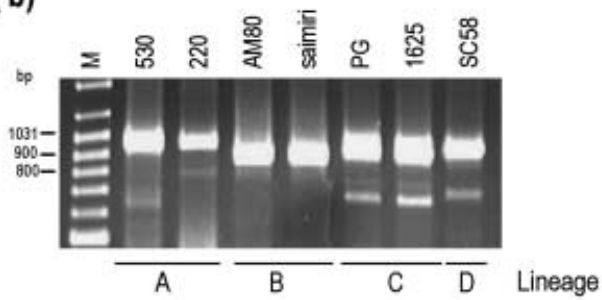

(e)

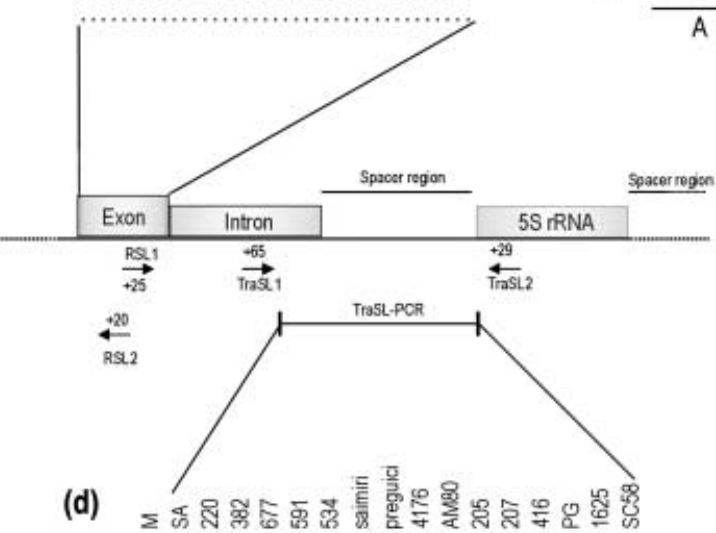

\section{$5^{\prime} \rightarrow 3^{\prime}$}

RSL1. TTCTGTACTATATTGGTATG

RSL2 - OCAATATAGTACAGAAACTG

TrRSL1 - GAACGGTOGTGTTCTG

TraSt.2 - GACGGGATGTGGTGC

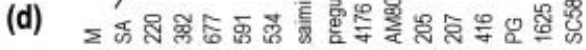

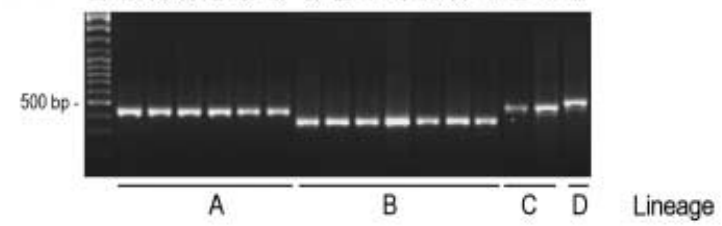

Lineage

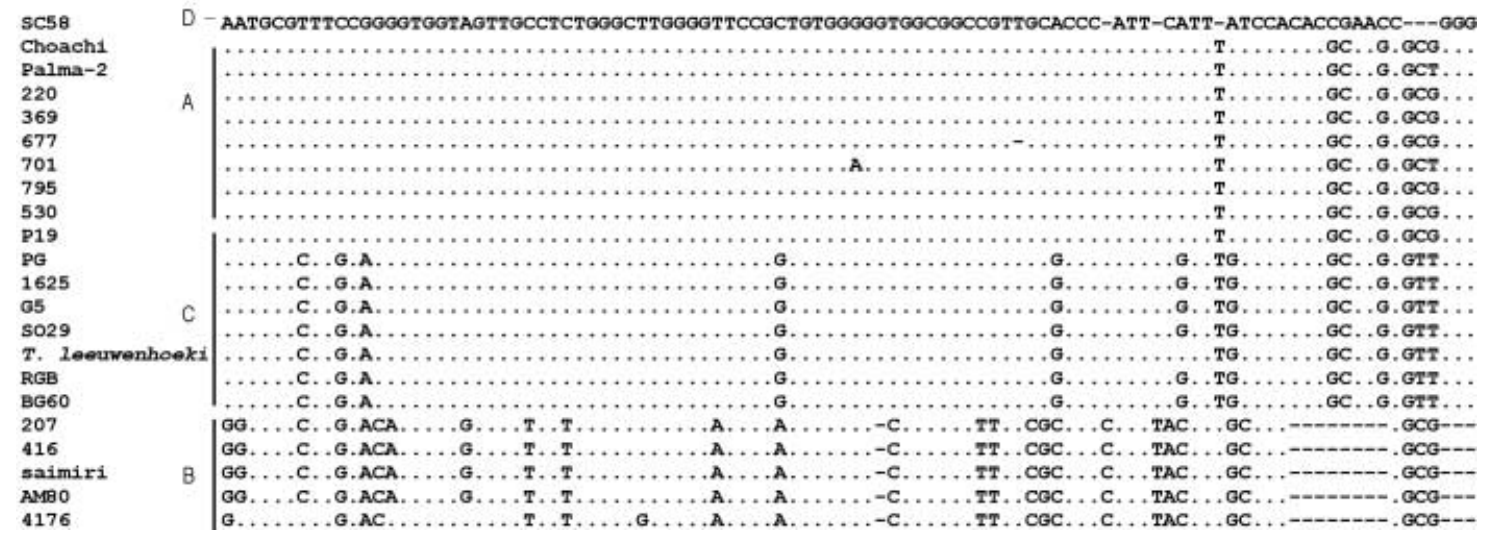

Fig. 1 (a) Alignment of the SL exon sequences from Trypanosoma rangeli isolates; (b) Agarose gel showing amplified fragments corresponding to whole SL repeat units of $T$. rangeli isolates stained with ethidium bromide; (c) Schematic diagram of the SL gene showing the annealing sites for oligonucleotides used as primers (in the box) used for PCR amplifications; (d) Agarose gel stained with ethidium bromide showing DNA fragments generated by TraSL-PCR using DNA from T. rangeli isolates of lineages A-D; (e) Alignment of SL intergenic spacer sequences of $T$. rangeli isolates from lineages A-D.

\section{SL repeat length and sequence polymorphism among T. rangeli isolates and allied species}

Full-length SL gene repeats of $T$. rangeli isolates amplified by PCR, determined by agarose gel electrophoresis (Fig. 1b) and confirmed by hybridization with SL exon-derived probe SL201 (data not shown), revealed variable repeat lengths compatible with those previously described for $T$. rangeli isolates. Figure 1a illustrates the size polymorphism of SL repeats within T. rangeli: (i) isolates from lineage A (530 and 220) had $~ 980-b p$ SL repeats, similar to previously described for T. rangeli SA (Murthy et al. 1992); (ii) isolates belonging to lineage B, represented by the isolates AM80, saimiri, preguici and legeri from wild mammals (Maia da Silva 
et al. 2004a, b), showed SL repeats of $\sim 840 \mathrm{bp}$; and (iii) members of lineage C, PG, 1625, T. leeuwenhoeki, present a SL repeat from $\sim 920-955 \mathrm{bp}$, as previously reported (Stevens et al. 1999; Gibson et al. 2000). The isolate SC58 (lineage D) had a repeat length of $\sim 915 \mathrm{bp}$ (Grisard et al. 1999). Length variability of SL repeats in T. rangeli isolates is due insertion/ deletion events and microsatellite repeats in the intergenic spacer (Fig. 1e).

Preliminary analysis of SL sequences from a small number of $T$. rangeli isolates suggested that this marker could also generate the same four lineages derived from RAPD and ribosomal markers (Maia da Silva et al. 1999). In this study, sequences of whole $\mathrm{SL}$ repeat units were determined for $T$. rangeli isolates AM80, saimiri, preguici and legeri (lineage $B)$, and aligned with sequences from isolates of other lineages (GenBank): A (SA, H8GS); C (RGB, T. leeuwenhoeki) and D (SC58), plus the sequence of BG60 from Costa Rica (here ascribed to lineage $C$ ) (Table 1 ). In contrast to the high degree of conservation of the exon sequences among trypanosomatids, the aligned SL repeat sequences of $T$. rangeli isolates disclosed two different exon sequences showing minor polymorphism: one shared by lineages $\mathrm{A}, \mathrm{C}$ and $\mathrm{D}$, identical to that of Trypanosoma cruzi, and the other only present in lineage B (Fig. 1a). Alignment of full-length sequences of seven isolates from the four lineages of $T$. rangeli showed very similar intron sequences (average divergence was $\sim 2.2 \%$ ) and highly variable intergenic sequences (average divergence was $\sim 30 \%$ ). Contrasting to the exon and intron sequences, alignment of intergenic sequences was unreliable due to the very high polymorphism (Fig. 1e).

\section{Genetic relationships between T. rangeli isolates based on} intergenic spacer sequences

To better evaluate the intraspecific variability and genetic relationships among $T$. rangeli lineages, we selected a moderately conserved region within the SL repeat to compare several isolates (Fig. 1c). With this purpose, we sequenced amplified fragments (380-500 bp) (Fig. 1d) of intergenic sequences flanked by the SL intron and 5SrRNA sequences of 21 further isolates (Table 1) and aligned them with the sequences reported above. The SL intergenic spacer in trypanosomatids has very high interspecies variability, precluding reliable alignments of $T$. rangeli sequences even with the closely related T. cruzi. Based on a similarity matrix constructed using aligned intergenic sequences (Fig. 1e depicts a part of this region to illustrate lineage-specific polymorphisms), it was possible to define four groups of T. rangeli. According to the phylograms constructed using the MP (data not shown) or ML (Fig. 2a) methods, the same four lineages (A-D) previously determined by RAPD and ribosomal gene (Maia da Silva et al. 2004a, b) were also defined by SL sequences. Lineage A, constituted by isolates from Venezuela, Colombia, Guatemala and the Brazilian Amazonia, had $~ 98 \%$ sequence similarity; lineage B, comprising exclusively isolates from the Brazilian Amazonia, showed the most significant sequence divergence ( $\sim 93$ 99\% similarity); and lineage C, composed by isolates from Panama, Colombia, Costa Rica and El Salvador, which shared $\sim 95 \%$ sequence similarity. Lineage B was separated for largest sequence divergences from all other lineages: (a) SL gene

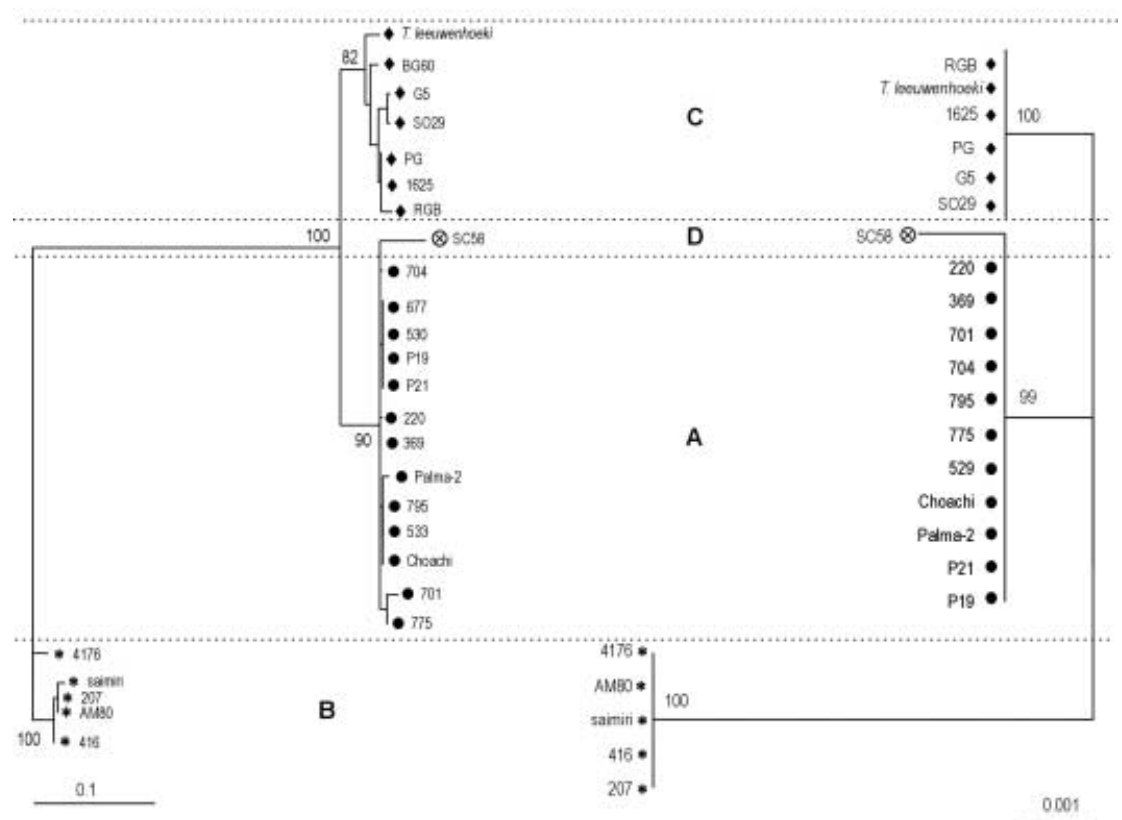

Fig. 2 Parity between phylogenetic relationships among Trypanosoma rangeli from lineages $\mathrm{A}(\bullet), \mathrm{B}(*), \mathrm{C}(\bullet)$ and $\mathrm{D}(\otimes)$ based on SL intergenic spacer sequences (a) and V7-V8 regions of SSU rRNA sequences (b), inferred by maximum likelihood (ML). For SL sequences alignment $(-\ln L=1343.1262)$, the best-fit evolutionary model, as determined by MODELTEST, was K80 (Kimura 1980 ) with gamma distribution $(\alpha=0.5505)$. For alignment of V7-V8 SSU rRNA sequences (- $\ln \mathrm{L}=1277.6781)$ the chosen model was K80. The numbers at nodes correspond to percentage of ML bootstrap support values derived from 100 replicates. 
A $(\sim 35 \%), C(41.5 \%)$ and D $(37 \%)$, whereas the smallest divergence separated lineage $C$ from $A(\sim 16 \%)$ and $\mathrm{D}(\sim 21 \%)$. Lineage D, represented by the isolate SC58 from southern Brazil, although separated by relatively small divergence from either lineages A $(\sim 16 \%)$ or $C(\sim 21 \%)$, were positioned together with lineage A (Fig. 2a).

\section{Parity analysis between lineages of T. rangeli defined based on spliced-leader and SSU rRNA sequences}

We previously demonstrated that SSU and ITS ribosomal sequences showed the same branching pattern of isolates within $T$. rangeli, with consistent heterogeneity segregating the isolates into four well-supported lineages (Maia da Silva et al. 2004b). To compare phylogenies of T. rangeli isolates using sequences from different genes, showing different evolutionary rates, we compare phylograms generated using SSUrRNA and intergenic SL sequences. The results showed total agreement between data from these two genes (Fig. 2).

\section{Phylogeography and evolutionary relationships between} T. rangeli lineages and Rhodnius spp.

Our data suggest an association between T. rangeli lineages and both geographical origin and complexes of Rhodnius. The geographical distribution of sylvatic species of Rhodniini varies from sylvatic species tightly associated with ecological traits of specific palms, like $R$. brethesi with the piassaba palm, to the most widespread $R$. prolixus, which besides sylvatic is domiciled in many areas (Fig. 3). The triatomines from which $T$. rangeli was isolated were classified according to morphological and molecular taxonomy. Cytochrome $b$ sequences from specimens of these triatomines were compared with sequences from GenBank. Brazilian triatomines were classified as $R$. robustus II (Rondonia) or R. brethesi (Rio Negro, Amazonas), specimens from Venezuela were classified as R. prolixus (Barinas and Trujillo) or R. robustus I (Trujillo) and those from Panama as R. pallescens (Table 1). Rhodnius species were confirmed by a phylogenetic analysis (Fig. 3a), that included sequences described in Monteiro et al. $(2000,2003)$.

By comparing the phylogeny of Rhodnius species (Fig. 3a) with that inferred for T. rangeli isolates (Fig. 2) and by comparing the geographical range of the isolates (Fig. 3b), we showed that there is total concordance of lineages and vector species and a very significant overlap in the distribution of Rhodnius species and T. rangeli lineages. The three major lineages analysed in this study could be related to the ecogeographical structure of the Rhodniini population as follows: (i) lineage A, circulating in Venezuela, Colombia, Guatemala, Honduras and Brazil, related to both domestic and sylvatic cycles of species of the $R$. prolixus complex; (iii) lineage $B$, occurring in the northern Brazilian Amazon region and related to sylvatic $R$. brethesi; and (iii) lineage $\mathrm{C}$, related to domestic and sylvatic cycles of the $R$. pallescens complex circulating in Panama, Costa Rica and Colombia. Accordingly, T. rangeli isolates from Rhodnius spp. from different complexes did not cluster together (Fig. 3; Table 1).

Comparison of Rhodnius and $T$. rangeli phylogenies revealed a total concordance of terminal taxa between $T$. rangeli lineages and complexes of their respective vector species, suggesting a long history of codivergence in these

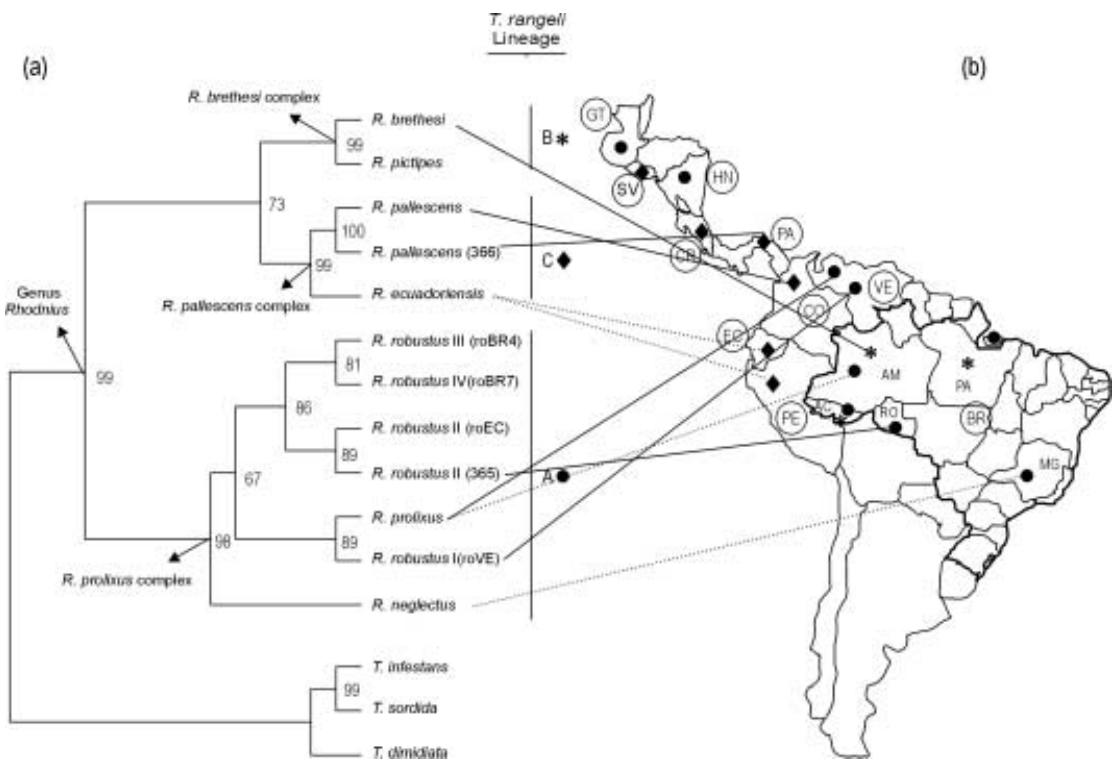

Fig. 3 Schematic representation linking the (a) phylogeography of Rhodnius species with (b) geographical range of Trypanosoma rangeli isolates from Central and South America (Table 1) ascribed to lineages A $(\bullet)$, B $(*)$ or C $(\bullet)$. (a) Phylogeography of Rhodnius was based on mitochondrial cyt $b$ sequences and inferred using maximum parsimony. The continuous lines indicate the collecting location of the specimens of Rhodnius spp. from which we obtained the T. rangeli isolates. Sequences from the isolates and from their respective Rhodnius hosts of origin were included in the phylogenetic analysis. Dotted lines indicate data from other studies. The numbers at nodes correspond to bootstrap values derived from 100 replicates. GT, Guatemala; SV, El Salvador; HN, Honduras; CR, Costa Rica; PA, Panama; $\mathrm{CO}$, Colombia; VE, Venezuela; BR, Brazil. States of Brazil: AM, Amazonas; AC, Acre; RO, Rondonia, PA, Para; MG, Minas Gerais. 
host-parasite assemblages. However, comparison of Rhodnius and T. rangeli phylogenies revealed incongruent branching patterns (Fig. 3) suggesting association between $T$. rangeli lineages and Rhodnius spp. by evolutionary process other than co-evolution. In the Rhodnius phylogeny based on cyt $b, R$. pallescens and $R$. brethesi complexes are sister groups (Fig. 3) whereas in the phylogeny of T. rangeli, lineage $\mathrm{C}(R$. pallescens complex) is sister to lineage A $(R$. prolixus complex) and not to lineage B (R. brethesi), which was distantly positioned (Fig. 2). Analysis done using TREEMAP2.02 program (not shown) suggested that host switching, sympatric speciation (duplication) and episodes of particular extinction (sorting) play a role in the evolutionary history of these host-parasite relationships.

\section{Identification and genotyping of T. rangeli isolates by TraSL-PCR based on SL intergenic sequences}

Aiming to define SL sequences that are able simultaneously to identify and genotype $T$. rangeli isolates without DNA sequencing, we developed the TraSL-PCR assay (Fig. 1d). The standardized PCR yielded DNA bands for all $58 \mathrm{~T}$. rangeli isolates examined. Specificity of TraSL-PCR was confirmed by negative results obtained using phylogenetically related organisms and/or flagellates sharing the same vertebrate and invertebrate hosts, such as T. cruzi (T. cruzi I, T. cruzi II and Z3 lineages), T. conorhini and Blastocrithidia spp. (data not shown). Besides high specificity, TraSL-PCR was shown to be very sensitive, detecting down to 500 pg of DNA by ethidium bromide staining and down to $100 \mathrm{pg}$ of DNA ( 10-20 cells) after hybridization using the SL amplified fragment of $T$. rangeli (SA) as probe (data not shown). The TraSL-PCR amplified fragments varied in length according to the lineage of the isolate whereas bands of the same length were shared by all isolates within each lineage: A (417 bp); B (380 bp); C (480 bp) and D (500 bp) (Table 1). All 58 isolates tested could be assigned to one of the four previously defined lineages (Fig. 1; Table 1).

\section{Discussion}

Studies regarding biological and molecular features of Trypanosoma rangeli isolates from distinct hosts and geographical origins have shown that they form a monophyletic group more closely related to the Schizotrypanum species (Trypanosoma cruzi) than to any other trypanosome (Stevens et al. 1999; Maia da Silva et al. 2004b). Despite being very closely related, several molecular markers separated $T$. rangeli isolates into distinct lineages, apparently related to Rhodnius complexes (Vallejo et al. 2002, 2003; Maia da Silva et al. 2004a, b; Urrea et al. 2005). To better understand the phylogenetic relationships within T. rangeli, we analysed $\mathrm{SL}$ and ribosomal sequences from $36 \mathrm{~T}$. rangeli isolates from distinct mammals plus 23 isolates from triatomine bugs.
Results from this study showed that all T. rangel $i$ isolates, from Central and South America, could be genotyped using PCR assays based on either SL or ITSrDNA markers into one of the four previously reported phylogenetic lineages, supported by analyses using SSU and ITS rDNA sequences (Maia da Silva et al. 2004a, b). Parity between phylogenetic relationships using SSU and ITS rDNA sequences was also reported for lineages of $T$. theileri (Rodrigues et al. 2006) and T. vivax (Cortez et al. 2006). Congruence between SL and SSUrRNA relationships among T. rangeli lineages shown in this study supported four previously defined phylogenetic lineages (Maia da Silva et al. 2004a, b), and corroborated lineages association with complexes of Rhodnius (Vallejo et al. 2002, 2003; Maia da Silva et al. 2004a, b; Urrea et al. 2005). Parity between genotyping using rDNA and SL markers was also demonstrated for lineages of T. cruzi (Zingales et al. 1998; Brisse et al. 2001), T. vivax (Cortez et al. 2006) and T. theileri (A.C. Rodrigues, F. Maia da Silva, M. Campaner \& M.M.G. Teixeira, unpublished).

Molecular phylogenies based on different sequences have indicated three major complexes of species within Rhodnius associated with specific ecotopes and geographical areas (Gaunt \& Miles 2000; Monteiro et al. 2000, 2003). The $R$. brethesi complex is the most heterogeneous, has been reported in the Brazilian and Venezuelan Amazon regions, and comprises $R$. brethesi associated with piassaba palms, and $R$. pictipes with a broader distribution. The R. pallescens complex is the most homogeneous and has been reported in Panama and Costa Rica (Central America) and northwest region of South America. The R. prolixus complex comprises six species, besides genetically different populations ascribed to the same species: $R$. robustus from Venezuela ( $\mathrm{RbI}$ ) is more closely related to $R$. prolixus than to Brazilian $R$. robustus, which is distributed into three genetically and geographically separated populations: RbII (west), RbIII (north and center) and RbIV (north) (Monteiro et al. 2003).

According to data from this study, isolates ascribed to lineage A came from northwest South America (Venezuela and Colombia), Central America (Honduras and Guatemala) and Brazil (western and eastern Amazon region). This lineage was associated with the $R$. prolixus complex and includes isolates from $R$. prolixus, $R$. robustus I and $R$. robustus II, plus isolates from humans, dog, opossum and monkeys. The isolate SC58, from southern Brazil, was previously ascribed to lineage D based on RAPD patterns and on SSU and ITS rDNA sequences (Maia da Silva et al. 2004b), and took into account previous studies that separated isolates from this region from all others by isoenzymes, RAPD and SL markers (Steindel et al. 1991, 1994; Grisard et al. 1999; Maia da Silva et al. 1999, 2004a). T. rangeli in southern Brazil should be transmitted by R. domesticus (Steindel et al. 1994), which is positioned in a relatively distant branch within the $R$. prolixus complex (Monteiro et al. 2000; Hypsa et al. 
2002). This putative vector agrees with the positioning of the isolate SC58 closest to lineage A in this study, contrasting to kDaNA markers that grouped this isolate with isolates of the R. pallescens complex (lineage C) (Vallejo et al. 2003; Urrea et al. 2005). Corroborating the hypothesis that lineage $\mathrm{A}$ is associated with the $R$. prolixus complex, T. rangeli circulating in Didelphis marsupialis and $R$. neglectus in southeast Brazil (Ramirez et al. 2002) were positioned within lineage $\mathrm{A}$ in this study, and also associated with $R$. prolixus using kDaNA markers (Gurgel-Goncalves et al. 2004).

Group B includes exclusively Brazilian isolates from humans, triatomines and wild mammals from the Brazilian Amazon region. Previous studies have shown that $R$. brethesi is the only vector for T. rangeli in the Rio Negro region of the northern Brazilian Amazon (Coura et al. 1996, 2002), and that isolates from $R$. brethesi can be identical to isolates from humans and monkeys (Maia da Silva et al. 2004a, b). The alleged absence of $R$. brethesi and piassava palms in areas of western (Acre) and eastern (Para) Amazon region where isolates ascribed to lineage $\mathrm{B}$ originate strongly suggests that $R$. pictipes, which is widespread and the closest relative species of $R$. brethesi, could, in addition to $R$. brethesi, be a vector of $T$. rangeli lineage $B$. We are currently trying to isolate $T$. rangeli from $R$. pictipes aiming to clarify this hypothesis.

Group C was formed by isolates from $R$. pallescens from Colombia and Panama, human isolates from Central America (Panama, Costa Rica and El Salvador), a dog isolate from Colombia and T. leeuwenhoeki from a sloth from Panama. Therefore, all isolates of this lineage came from within the ecogeographical range of the $R$. pallescens complex. In agreement with data using SL genes, these isolates had been previously grouped and associated with the R. pallescens complex using RAPD and SSU and ITSrDNA (Maia da Silva et al. 2004a, b). Associations between T. rangeli isolates from Colombia, Panama and Peru and R. pallescens complex were also established by kDNA and SL (mini-exon) markers (Vallejo et al. 2003; Urrea et al. 2005).

The relative patterns of $T$. rangeli grouping could be explained by the geographical structure of Rhodnius spp. In conformity with the distribution of $R$. robustus and $R$. brethesi, $T$. rangeli isolates from the Brazilian Amazon were segregated into lineages A and B. In general, isolates from the same geographical regions are more closely related, excepting for isolates from regions where different Rhodnius complexes naturally overlapped, or shared the same habitats after their human introduction. For example, isolates from the same area of Colombia were segregated into lineages $\mathrm{A}$ and $\mathrm{C}$ related to the $R$. prolixus or $R$. pallescens complexes, respectively (Vallejo et al. 2002, 2003; Urrea et al. 2005).

Complex vector-parasite interactions reflect a long evolutionary history of $T$. rangeli lineages with different Rhodnius complex. The congruency among ribosomal, SL, $\mathrm{RAPD}$ and $\mathrm{kDaNA}$ markers suggested that $T$. rangeli line- ages evolved with a nonrandom association of independent molecular markers, pointing towards the existence of linkage disequilibrium and the absence of gene flow between recently diverged lineages. In this study, all T. rangeli lineages contain isolates from different host species (humans and domestic or sylvatic mammals), thus without association with particular taxon of vertebrate. The small divergence separating $T$. rangeli lineages is compatible with a recent divergence of Rhodnius species (Monteiro et al. 2003). In contrast, large genetic distances separate T. cruzi lineages that appear to evolve in association with their preferential mammalian hosts, which diverged a long time ago (Gaunt \& Miles 2000; Yeo et al. 2005). Contrasting to vertebrate host-restriction reported for some trypanosomes of rabbit (Hamilton et al. 2005), bats (Stevens et al. 2001) and artiodactyls (Rodrigues et al. 2003, 2006), avian trypanosomes apparently lack vertebrate-host specificity (Sehgal et al. 2001) likewise T. cruzi and T. rangeli.

Demonstration that $T$. rangeli isolates from distinct Rhodnius complexes living in sympatry are separated in distinct lineages suggests evolution of $T$. rangeli lineages by a long coexistence with their sympatric vectors in independent transmission cycles. Phylogeographical analysis strongly supports an association between geographical regions and both $T$. rangeli lineages and Rhodnius spp. Therefore, biogeography is very important in structuring these host-parasite assemblages.

Data from this study demonstrated total concordance between different $T$. rangeli lineages and the complexes of Rhodnius using either SL or ribosomal markers, which suggests vector-parasite co-evolution. However, comparison of Rhodnius and T. rangeli phylogenies showed incongruent branching patterns, which indicates a lack of or little cospeciation, with host-switching indicating that the evolutionary histories of Rhodnius and $T$. rangeli are complex. In our analysis, $T$. rangeli lineage $C$ (associated to $R$. pallescens) was more closely related to lineage A ( $R$. prolixus) than to lineage $\mathrm{B}$ (R. brethesi), in disagreement with the close relationship of $R$. pallescens and $R$. brethesi complexes based on cyt $b$ phylogeny (Monteiro et al. 2000, 2003). A phylogeny of the tribe Rhodniini based on zymodemes suggested a basal group comprising $R$. pictipes and $R$. brethesi originated in northern Amazonia and then dispersed northwest and south to give rise to the $R$. pallescens and $R$. prolixus complexes (Dujardin et al. 1999). However, R. pictipes was not found as the most primitive branch in other studies. The phylogeny based on the $16 \mathrm{~S}$ mitochondrial rDNA positioned R. pictipes / $R$. brethesi closer to $R$. prolixus than to $R$. pallescens, which was positioned in the most basal branch of the Rhodniini (Hypsa et al. 2002). Therefore, depending on methods, sequences and parameters used, different topologies were obtained, placing the $R$. pictipes complex as sister group of either the R. pallescens or the R. prolixus complex (Lyman et al. 1999; Monteiro et al. 2000; Hypsa et al. 2002). A recent 
phylogenetic interpretation of $16 \mathrm{~S}$ mtrDNA sequence data with regard to the geographical distribution of Rhodnius spp. and geological events affecting the origin and diversification of Rhodniini support a complex biogeographical history of this tribe (Paula et al. 2006).

Apparently, a major role in the evolutionary origin of $T$. rangeli lineages is their geographical distribution and specific ecotopes and niches of Rhodnius species. However, the evolutionary history of Rhodniini is far from being clearly understood and the vector-parasite associations found in the present study must be interpreted with caution. A better understanding of population structure and the factors involved in lineage segregation within $T$. rangeli requires analysis of a larger number of isolates from invertebrate and vertebrate hosts from a large ecogeographical range, together with a better phylogeographical analysis of Rhodnius. More lineages can be described by using other molecular markers and by analysing other Rhodnius spp. from new geographical areas. The $T$. rangeli-specific SL-derived PCR assay that we have developed may be useful for larger surveys by allowing simultaneous identification and genotyping of $T$. rangeli.

\section{Acknowledgements}

We are grateful to Dr Erney P. Camargo and Dr Jeffrey J. Shaw for their continued encouragement, discussions and constructive criticisms in reviewing our manuscript. We thank M. C. Brigido and C. Bonvicino for blood samples and identification of monkeys, and Toby Barret and Jeffrey J. Shaw for T. rangeli isolates. We thank Robson C. Ferreira and Myrna G. Serrano for initial help in the SL gene sequencing. We are indebted to several collaborators and students for their inestimable help in the fieldwork and to anonymous reviewers for valuable comments and English revision that improved our manuscript. This project was supported by Brazilian grants of CNPq, FAPESP and PRONEX. Adriana C. Rodrigues and Flávia Maia da Silva are postdoctoral fellows sponsored by $\mathrm{CNPq}$ and CAPES, respectively.

\section{References}

Aksoy S, Shay GL, Villanueva MS, Beard CB, Richards FF (1992) Spliced leader RNA sequences of Trypanosoma rangeli are organized within the 5S rRNA-encoding genes. Gene, 113, 239-243.

Aljanabi SM, Martinez I (1997) Universal and rapid salt-extraction of high quality genomic DNA for PCR-based techniques. Nucleic Acids Research, 25, 4692-4693.

Añez N (1984) Studies on Trypanosoma rangeli Tejera, 1920. VII. Its effect on the survival of infected triatomine bugs. Memórias do Instituto Oswaldo Cruz, 79, 249-255.

Banks JC, Paterson AM (2005) Multi-host parasite species in cophylogenetic studies. International Journal for Parasitology, 35, 741-746.

Brisse S, Verhoef J, Tibayrenc M (2001) Characterisation of large and small subunit rRNA and mini-exon genes further supports the distinction of six Trypanosoma cruzi lineages. International Journal for Parasitology, 31, 1218-1226.
Brooks AL, Ferrao DR (2005) The historical biogeography of coevolution: emerging infectious diseases are evolutionary accidents waiting to happen. Journal of Biogeography, 32, 1291-1299.

Cortez AP, Ventura RM, Rodrigues AC et al. (2006) The taxonomic and phylogenetic relationships of Trypanosoma vivax from South America and Africa. Parasitology, 133, 159-169.

Coura JR, Fernandes O, Arboleda M et al. (1996) Human infection by Trypanosoma rangeli in the Brazilian Amazon. Transactions of the Royal Society of Tropical Medicine and Hygiene, 90, 278279.

Coura JR, Junqueira AC, Fernandes O, Valente SA, Miles MA (2002) Emerging Chagas disease in Amazonian Brazil. Trends in Parasitology, 18, 171-176.

Cuervo C, Lopez MC, Puerta C (2006) The Trypanosoma rangeli histone $\mathrm{H} 2 \mathrm{~A}$ gene sequence serves as a differential marker for KP1 strains. Infection, Genetic and Evolution, 6, 401-409.

D'Alessandro A, Saravia NG (1999) Trypanosoma rangeli. In: Protozoal Diseases (ed. Gilles HM), pp. 398-412. Arnold, London.

De Stefani Marquez D, Rodrigues-Ottaiano C, Monica Oliveira R et al. (2006) Susceptibility of different triatomine species to Trypanosoma rangeli experimental infection. Vector Borne Zoonotic Disease, 6, 50-56.

Dujardin JP, Garcia-Zapata MT, Jurberg J et al. (1991) Which species of Rhodnius is invading houses in Brazil? Transactions of the Royal Society of Tropical Medicine and Hygiene, 85, 679680.

Dujardin JP, Chavez T, Moreno JM et al. (1999) Comparison of isoenzyme electrophoresis and morphometric analysis for phylogenetic reconstruction of the Rhodniini (Hemiptera: Reduviidae: Triatominae). Journal of Medical Entomology, 36, 653-659.

Galvão C, Carcavallo R, Rocha DS, Jurberg J (2003) A checklist of the current valid species of the subfamily Triatominae Jeannel, 1919 (Hemiptera, Reduviidae) and their geographical distribution, with nomenclatural and taxonomic notes. Zootaxa, 202, $1-36$.

Gaunt M, Miles M (2000) The ecotopes and evolution of triatomine bugs (triatominae) and their associated trypanosomes. Memórias do Instituto Oswaldo Cruz, 95, 557-565.

Gibson W, Bingle L, Blendeman W et al. (2000) Structure and sequence variation of the trypanosome spliced leader transcript. Molecular and Biochemical Parasitology, 107, 269-277.

Grisard EC, Campbell DA, Romanha AJ (1999) Mini-exon gene sequence polymorphism among Trypanosoma rangeli strains isolated from distinct geographical regions. Parasitology, 118, 375-382.

Guhl F, Vallejo GA (2003) Trypanosoma (Herpetosoma) rangeli Tejera, 1920: an updated review. Memórias do Instituto Oswaldo Cruz, 98, 435-442.

Gurgel-Goncalves R, Ramalho ED, Duarte MA et al. (2004) Enzootic transmission of Trypanosoma cruzi and T. rangeli in the Federal District of Brazil. Revista do Instituto de Medicina Tropical de São Paulo, 46, 323-330.

Hamilton PB, Stevens JR, Holz P, Boag B, Cooke B, Gibson WC (2005) The inadvertent introduction in Australia of Trypanosoma nabiasi, the trypanosome of the European rabbit (Oryctolagus cuniculus), and its potencial for biocontrol. Molecular Ecology, 14, 3167-3175.

Huyse T, Poulin R, Theron A (2005) Speciation in parasites: a population genetics approach. Trends in Parasitology, 21, $469-475$. 
Hypsa V, Tietz DF, Zrzavy J et al. (2002) Phylogeny and biogeography of Triatominae (Hemiptera: Reduviidae): molecular evidence of a New World origin of the Asiatic clade. Molecular Phylogenetics and Evolution, 23, 447-457.

Kimura M (1980) A simple method for estimating evolutionary rates of base substitutions through comparative studies of nucleotide sequences. Journal of Molecular Evolution, 16, 111-120.

Lent H, Wygodzinsky P (1979) Revision of the Triatominae (Hemiptera: Reduviidae) and their significance as vectors of Chagas disease. Bulletin of the American Museum of Natural History, 163, 123-520.

Lyman DF, Monteiro FA, Escalante AA et al. (1999) Mitochondrial DNA sequence variation among triatomine vectors of Chagas' disease. American Journal of Tropical Medicine and Hygiene, 60, 377-386

Maia da Silva F, Rodrigues AC, Campaner M, Ferreira RC, Takeda GF, Teixeira MMG (1999) Trypanosoma (Herpetosoma) spp.: genetic diversity and taxonomic position defined by ribosomal, spliced leader and RAPD markers. Memórias do Instituto Oswaldo Cruz, 94 (Suppl. II), 158.

Maia da Silva F, Rodrigues AC, Campaner M et al. (2004a) Randomly amplified polymorphic DNA analysis of Trypanosoma rangeli and allied species from human, monkeys and other sylvatic mammals of the Brazilian Amazon disclosed a new group and a species-specific marker. Parasitology, 128, 283-294.

Maia da Silva F, Noyes H, Campaner M et al. (2004b) Phylogeny, taxonomy and grouping of Trypanosoma rangeli isolates from man, triatomines and sylvatic mammals from widespread geographical origin based on SSU and ITS ribosomal sequences. Parasitology, 129, 549-561.

Miles MA, Arias JR, Cedillos RA (1983) Vertebrates hosts and vectors of Trypanosoma rangeli in the Amazon basin of Brazil. American Journal of Tropical Medicine and Hygiene, 32, 1251-1259.

Monteiro FA, Wesson DM, Dotson EM, Schofield CJ, Beard CB (2000) Phylogeny and molecular taxonomy of the Rhodniini derived from mitochondrial and nuclear DNA sequences. American Journal of Tropical Medicine and Hygiene, 62, 460-465.

Monteiro FA, Barrett TV, Fitzpatrick S et al. (2003) Molecular phylogeography of the Amazonian Chagas disease vectors Rhodnius prolixus and R. robustus. Molecular Ecology, 12, 9971006.

Murthy VK, Dibber KM, Campbell DA (1992) PCR amplification of mini-exon genes differentiates Trypanosoma cruzi from Trypanosoma rangeli. Molecular and Cellular Probes, 6, 237-243.

Page RDM (1991) Clocks, clades and cospeciation: comparing rates of evolution and timing of cospeciation events in hostparasite assemblage. Systematic Zoology, 40, 188-198.

Page RDM, Charleston MA (1998) Trees within trees: phylogeny and historical associations. Trends in Ecology \& Evolution, 13, 356-359.

Paterson AM, Banks J (2001) Analytical approaches to measuring cospeciation of host and parasites: through a glass, darkly. International Journal for Parasitology, 31, 1012-1022.

Paula AS, Diotaiuti L, Galvão C (2006) Systematic and biogeography of Rhodiniini (Heteroptera: Redividae: Triatominae) based on $16 \mathrm{~S}$ mitocondrial rDNA sequences. Journal of Biogeography, 34, 699-712.

Posada D, Crandall KA (1998) Modeltest: testing the model of DNA substitution. Bioinformatics, 14, 817-818.
Ramirez LE, Lages-Silva E, Alvarenga-Franco F et al. (2002) High prevalence of Trypanosoma rangeli and Trypanosoma cruzi in opossums and triatomids in a formerly-endemic area of Chagas disease in Southeast Brazil. Acta Tropica, 84, 189-198.

Rodrigues AC, Campaner M, Takata CS et al. (2003) Brazilian isolates of Trypanosoma (Megatrypanum) theileri: diagnosis and differentiation of isolates from cattle and water buffalo based on biological characteristics and randomly amplified DNA sequences. Veterinary Parasitology, 116, 185-207.

Rodrigues AC, Paiva F, Campaner M, Stevens JR, Noyes HA, Teixeira MMG (2006) Phylogeny of Trypanosoma (Megatrypanum) theileri and related trypanosomes reveals lineages of isolates associated with artiodactyl hosts diverging on SSU and ITS ribosomal sequences. Parasitology, 3, 1-10.

Sehgal RNM, Jones HI, Smith TB (2001) Host specificity and incidence of Trypanosoma in some African rainforest birds: a molecular approach. Molecular Ecology, 10, 2319-2327.

Serrano MG, Nunes LR, Campaner M, Buck GA, Camargo EP, Teixeira MMG (1999) Trypanosomatidae: phytomonas detection in plants and phytophagous insects by PCR amplification of a genus-specific sequence of the spliced leader gene. Experimental Parasitology, 9, 268-279.

Souto RP, Fernandes O, Macedo AM, Campbell DA, Zingales B (1996) DNA markers define two major phylogenetic lineages of Trypanosoma cruzi. Molecular and Biochemical Parasitology, 83, 141-152.

Steindel M, Carvalho Pinto JC, Toma HK, Mangia RHR, Ribeiro-Rodrigues R, Romanha AJ (1991) Trypanosoma rangeli (Tejera, 1920) isolated from sylvatic rodent (Echimys dasytrix) in Santa Catarina island, Santa Catarina state: first report of this trypanosome in Southern Brazility. Memórias do Instituto Oswaldo Cruz, 86, 73-79.

Steindel M, Dias Neto E, Pinto CJ et al. (1994) Randomly amplified polymorphic DNA (RAPD) and isoenzyme analysis of Trypanosoma rangeli strains. Journal of Eukaryotic Microbiology, 41, 261-267.

Stevens JR, Teixeira MMG, Bingle LE, Gibson WC (1999) The taxonomic position and evolutionary relationships of Trypanosoma rangeli. International Journal for Parasitology, 29, 749-757.

Stevens JR, Noyes HA, Schofield CJ, Gibson W (2001) The molecular evolution of Trypanosomatidae. Advances in Parasitology, 48, 1-56.

Strimmer KN, Von Haeseler A (1996) Quartet puzzling: a quartet maximum likelihood method for reconstructing tree topologies. Molecular Biology and Evolution, 13, 964-969.

Swofford DL (2002) PAUP*: Phylogenetic Analysis Using Parsimony (*and Other Methods), Version 4.0b10. Sinauer Associates, Sunderland, Massachusetts.

Teixeira MMG, Serrano MG, Nunes LR et al. (1996) Trypanosomatidae: a spliced-leader-derived probe specific for the genus Phytomonas. Experimental Parasitology, 84, 311-319.

Urrea DA, Carranza JC, Cuba CAet al. (2005) Molecular characterisation of Trypanosoma rangeli strains isolated from Rhodnius ecuadoriensis in Peru, R. colombiensis in Colombia and R. pallescens in Panama, supports a co-evolutionary association between parasites and vectors. Infection, Genetic and Evolution, 5, 123129.

Vallejo GA, Guhl F, Carranza JC et al. (2002) kDNA markers define two major Trypanosoma rangeli lineages in Latin America. Acta Tropica, 81, 77-82. 
Vallejo GA, Guhl F, Carranza JC et al. (2003) Parity between kinetoplast DNA and mini-exon gene sequences supports either clonal evolution or speciation in Trypanosoma rangeli strains isolated from Rhodnius colombiensis, R. pallescens and R. prolixus in Colombia. Infection, Genetic and Evolution, 3, 39-45.

Ventura RM, Paiva F, Silva RA et al. (2001) Trypanosoma vivax: characterization of the spliced-leader gene of a Brazilian stock and species-specific detection by PCR amplification of an intergenic spacer sequence. Experimental Parasitology, 99, 37-48.

Yeo M, Acosta N, Llewellyn M, Sánchez et al. (2005) Origins of Chagas disease: Didelphys species are natural hosts of Trypanosoma cruzi I and armadillos hosts of Trypanosoma cruzi II, including hybrids. International Journal for Parasitology, 35, 225-233.

Zingales B, Souto RP, Mangia RH et al. (1998) Molecular epidemiology of American trypanosomiasis in Brazil based on dimorphisms of rRNA and mini-exon gene sequences. International Journal for Parasitology, 28, 105-112.
Flávia Maia da Silva and Angela C. V. Junqueira completed this work as part of their PhD thesis. Together with Adriana C. Rodrigues, they are currently postgraduate students working on biodiversity of trypanosomes, processes structuring biodiversity, and evolutionary history of Trypanosoma. Marta Campaner works as biologist at the University of São Paulo where she is responsible for the isolation and culture of trypanosomes. Gladys Crisante and Zuleima C. E. Caballero are graduate students and collaborated on fieldwork during this study. Fernando Monteiro is a research scientist interested in the systematics, phylogeography and population genetics of insect vectors of parasitic diseases. Professors Marta M. G. Teixeira, Luis E. Ramirez, José Rodrigues Coura (Brazil) and Nestor Añez (Venezuela) have a longstanding interest and experience in different aspects of trypanosome infection on humans and triatomine bugs, including transmission and disease emergence in Amazonia. Marta Teixeira is also interested in diversity, phylogeny, biogeography and phylogeography, molecular diagnosis and epidemiology of trypanosomes from all vertebrate classes and their insect vectors. 